Original Research Article

\title{
Adequacy of pain management in oncology patients at a tertiary hospital in Ghana
}

\author{
Akua A. Abruquah ${ }^{1}$, Robert P. Biney ${ }^{2}$, Ernest B. Osei-Bonsu ${ }^{3}$, Kofi M. Boamah $^{3}$, Eric Woode ${ }^{4}$
}

${ }^{1}$ Department of Pharmaceutical Sciences, Kumasi Technical University, Kumasi, Ghana ${ }^{2}$ Department of Pharmacology, School of Medical Sciences, University of Cape Coast, Cape Coast, Ghana

${ }^{3}$ Oncology Directorate, Komfo Anokye Teaching Hospital, Kumasi, Ghana

${ }^{4}$ Department of Pharmacology, College of Health Sciences,

Kwame Nkrumah University of Science and Technology,

Kumasi, Ghana

Received: 21 November 2016

Accepted: 24 December 2016

\section{*Correspondence to:}

Mrs. Akua Afriyie Abruquah, Email:

akua.abruquah@gmail.com

Copyright: () the author(s), publisher and licensee Medip Academy. This is an openaccess article distributed under the terms of the Creative Commons Attribution NonCommercial License, which permits unrestricted noncommercial use, distribution, and reproduction in any medium, provided the original work is properly cited.

\section{ABSTRACT}

Background: Although cancer pain is well documented, efficient management is largely inadequate in most patients especially in developing countries. This study evaluated the adequacy of pain management as well as potential social factors that may be associated with inadequate pain management.

Methods: 204 ambulatory oncology patients (82\% female; mean age 53.5) attending clinic at the Oncology Directorate, of a tertiary hospital in Ghana from January to December, 2015 were recruited and their pain severity and functional interference assessed with the Brief Pain Inventory (BPI). The adequacy of pain management was computed as the pain management index (PMI) using the BPI.

Results: Although $62 \%$ of respondents were prescribed high potency opioids, $56.9 \%$ of them exhibited significant pain while $34.9 \%$ required a stronger analgesic to manage their pain. Majority of patients $(56 \%)$ were over-managed for their pain (had PMI score >0). Only 26.4\% had optimal cancer pain management. Pain interfered mostly with patients' sleep $(46.2 \%)$ and general activity $(42.5 \%)$. Patients with high pain intensity were more likely to have it affect their daily activity $(\mathrm{P}<0.0001)$. Men were more likely to have inadequate pain management than females.

Conclusions: This study has shown that whereas pain management may be adequate, there is the tendency for opioid tolerance and abuse as over $50 \%$ of patients receive more analgesics than required. The tenets of the WHO 3-step analgesic ladder should be strictly adhered to achieve optimum cancer pain relief.

Keywords: Analgesics, Brief pain inventory, Cancer pain, Pain management index

suffered by cancer patients. $^{2}$ Cancer pain is multidimensional and multifaceted and can have detrimental effects on the functioning of patients and adversely affect their quality of life. ${ }^{3,4}$

Suboptimal cancer pain control can be very debilitating. The World Health Organization (WHO) in 1986 published the Cancer Pain Relief report which 
emphasized the right of patients to optimal pain management with effective pain treatment modalities. ${ }^{[5]}$ In spite of this however, $50-80 \%$ of cancer patients have poorly managed pain. ${ }^{5}$ It is estimated that one-third of all cancer patients and $60 \%-90 \%$ of patients with advanced cancer experience chronic or recurrent cancer related pain. ${ }^{4,6}$

Pain Management Index (PMI) is a well-validated and widely used method for assessing the adequacy of pain management developed by Cleeland. ${ }^{2}$ PMI is modelled on the concept of the cancer pain treatment guidelines established by the World Health Organization (WHO). ${ }^{7}$ Per the tenets of PMI, pain management is considered adequate when there is congruence between patient's subjective self-reported pain intensity and the prescribed analgesic(s).

Existing data on the extent of under-recognition and under-treatment of cancer pain is predominantly from studies in developed countries. ${ }^{1}$ Very little is known and documented about the extent of cancer pain assessment and management in developing countries such as Ghana. ${ }^{1,8}$ This paucity of information on the adequacy of cancer pain management from developing countries is a barrier to effective cancer pain management as existing pain treatment modalities are not race or culture sensitive. This study seeks to investigate the adequacy of cancer pain management using PMI in a tertiary oncology centre in Ghana.

\section{METHODS}

\section{Study design/ duration and setting}

The study was a hospital-based descriptive crosssectional study conducted from January to December, 2015. The study was conducted at the Oncology Directorate (a comprehensive cancer treatment facility which provides complete therapy for almost all types of cancer) of the Komfo Anokye Teaching Hospital (KATH) which is located in Kumasi, Ghana $\left(6^{\circ} 41^{\prime} 46.78^{\prime \prime} \mathrm{N}\right.$ and $\left.1^{\circ} 37^{\prime} 44.79^{\prime \prime} \mathrm{W}\right)$. KATH is the second largest hospital in Ghana. Due to the geographical location coupled with the extent of commercial activities in Kumasi, KATH serves patients from all the northern regions of Ghana, some parts of the middle belt and other counties in the West African sub-region and is considered as one of the best hospitals in the sub-region for the provision of cancer care.

\section{Ethical issues}

Ethical clearance was sought from the Committee on Human Research, Publications and Ethics (CHRPE), Kwame Nkrumah University of Science and Technology (KNUST), Kumasi-Ghana (CHRPE/RC/012/15). Permission to conduct the study was sought from the Oncology Directorate, KATH before commencement of data collection. Verbal informed consent was sought from patients prior to the conduction of interviews and the interviews were conducted in accordance with the Declaration of Helsinki for human research. ${ }^{2}$

\section{Sample size}

Patients recruited in this study were outpatients with different cancers attending clinic at the Oncology Directorate of KATH who had positive histological results. 747 patients were newly diagnosed with various neoplasms between January and December 2015. Of this, a sample size of 261 was calculated using the Yamene's formula:

$$
n=\frac{N}{1+N(e)^{2}}
$$

Where $\mathrm{n}=$ sample size, $\mathrm{N}=$ total population size and $\mathrm{e}=$ sampling error.

204 patients representing $78 \%$ of the computed sample size were interviewed with the Brief Pain Inventory questionnaire. The shortfall in the sample size is due to patient factors such as: observable cognitive disturbance, severe illness, lack of verbal informed consent, and inability to read or understand English or the native Twi language.

\section{Structured Interviews}

The principal researcher (who is bilingually competent in both Twi and English languages) approached all potentially eligible patients at the Oncology Directorate, KATH after pilot testing of the questionnaire. The interviews were conducted in Twi or English language depending on the level of education of the patient or the patient's preference. Twi is the local language of the indigenous people of the Ashanti Region of Ghana; which is spoken by about two-thirds of the population and understood by nearly all Ghanaians.

\section{Inclusion and Exclusion Criteria}

The study included cancer outpatients of either sex who were 18 years or older, had pathologic diagnosis of cancer (primary or metastatic) and could comprehend English or Twi language. Patients who were less than 18 years, had documented or observable psychiatric or neurological disorders (e.g., dementia or psychosis) and could not understand Twi language or read English language were excluded from the study. Patients who did not provide verbal informed consent were also excluded.

\section{Analgesic(s) prescribed and other medical information}

Patient information such as cancer type, site and stage as well as the type of prescribed analgesic drugs were obtained from the patients' medical records and from a hospital based electronic clinical management database. 


\section{Data Collection Instrument}

The Brief Pain Inventory (BPI)- an easily understood and scored Patient Reported Outcome (PRO) tool which can be self-administered or interviewer administered was used. ${ }^{6}$ Given that pain is multidimensional in nature, the BPI was designed to measure two key aspects of pain: sensory pain and reactive pain based on individual subjective self- report. ${ }^{4,5}$ The sensory aspect of pain, also known as pain intensity is measured by four items ("pain at its worst", "pain at its least", "pain on the average", and "pain now") of the BPI on an 11-point Numeric Rating Scale (NRS) $(0=$ "no pain" to $10=$ "severe or excruciating pain as bad as you can imagine"). Pain intensity is defined as self-perceived magnitude of pain at a given time interval.

The reactive aspect of pain; also known as functional interference is measured by seven items (general activity, mood, walking ability, normal work, relations with other people, sleep, and enjoyment of life) on the BPI using an 11-point NRS ranging from 0 which represents "does not interfere" to 10 which indicates "completely interferes". Functional interference is defined as the degree to which pain impacts various aspects of typical daily functioning.

\section{Data analysis and statistical methods}

Internal consistency of the test instrument was computed as the Cronbach's alpha coefficient as described by Cronbach. ${ }^{6}$ The Mann-Whitney, Kruskal-Wallis and independent $t$-tests were done where appropriate. Pain intensity was categorized as a score of $0=$ no pain, $1=$ mild pain (1-4), $2=$ moderate pain (5-6) and $3=$ severe pain (7-10) and the pain intensity index calculated by adding the scores of all the 4 pain intensity items., ${ }^{2,-11}$ Functional interference index was calculated by adding all the 7 functional interference items. ${ }^{10,12}$ An exploratory univariate analysis was used to determine the association between psychosocial factors and pain intensity as well as functional interference.

The PMI was calculated by subtracting the pain score (pain intensity) from the analgesic score. ${ }^{13}$ Analgesic scores of $0,1,2$, and 3 were assigned when patients were prescribed no pain medication, non-opioids, "weak" opioids, and "strong" opioids, respectively in accordance with the WHO's "analgesic ladder" approach to cancer pain management. A negative PMI score was considered an indicator of potential inadequate pain management. ${ }^{9,13}$

\section{RESULTS}

\section{Participant Characteristics}

A potential pool of 261 outpatients was identified for the study. The available data was obtained from 204 patients, whose sociodemographic and clinical characteristics are shown in Table 1. Female participants accounted for $82.9 \%$ of the sample; the mean age was 53.5 years (SD $\pm 15.5) .89 \%$ were Christians, $51.5 \%$ were married, $39.2 \%$ were unemployed, $81.9 \%$ had national health insurance, $9.1 \%$ had a positive family history of cancer. The most frequent cancer sites were breast $(37.7 \%)$, gynaecological $(26.9 \%)$ and oropharyngeal $(9.1 \%)$.

Table 1: Demographic and clinical characteristics of the study sample $(n=204)$.

\begin{tabular}{|llll|}
\hline Characteristic & & n & $\%$ \\
\hline Mean age \pm SD & $53.5 \pm 15.5$ & 204 & \\
\hline Gender & Female & 169 & 82.9 \\
\hline Educational level & Elementary/ JHS & 66 & 32.4 \\
\hline Marital status & Married & 105 & 51.5 \\
\hline Religion & Christianity & 178 & 89.0 \\
\hline Job status & Unemployed & 80 & 39.2 \\
\hline Family history & Positive & 17 & 9.1 \\
\hline Cancer site & Breast & 66 & 37.7 \\
\hline & Gynaecological & 47 & 26.9 \\
\hline & Oropharyngeal & 16 & 9.1 \\
\hline Cancer staging & Early (Stages 1 and 2) & 15 & 34.9 \\
\hline & $\begin{array}{l}\text { Advanced (Stages 3 } \\
\text { and 4) }\end{array}$ & 28 & 65.1 \\
\hline
\end{tabular}

\section{Internal consistency of data collection tool}

The Cronbach's $\alpha$ coefficient was computed to be 0.78 for pain intensity index (made up of 4 items) and 0.92 for functional interference index (made up of 7 items). The Cronbach's $\alpha$ coefficient for both pain intensity index and functional interference index composite scores were computed to be 0.88 which is acceptable. ${ }^{6}$

\section{Pain severity in this population}

Using a previously validated pain severity classification method by Okuyama et al, $28.4 \%$ of patients reported severe pain ( 7 or greater), $63.7 \%$ reported moderate pain $(5-6)$ and $7.8 \%$ reported mild pain (1-4) on the BPI. ${ }^{2}$

\section{Functional interference in sample population}

Based on respondents' responses, pain completely interfered (score $\geq 7$ ) with patients' sleep $(46.2 \%)$, general activity $(42.5 \%)$, walking ability $(40.4 \%)$ and $\operatorname{mood}$ $(30.5 \%)$. Complete interference on the rest of the items under functional interference was more than $20 \%$ but less than $30 \%$.

\section{Adequacy/Inadequacy of analgesic drug therapy}

Of the 204 participants, $50.5 \%$ were prescribed analgesics. $37.8 \%$ were receiving opioid analgesic treatment: $31.4 \%$ were taking strong opioids and $6.4 \%$ were taking weak opioids. The proportion of patients with negative PMI was $16 \%$, which indicated under treatment of pain and less-than-adequate analgesics based on the WHO guidelines. $26.4 \%$ had optimum PMI 
$(\mathrm{PMI}=0)$, and $57.5 \%$ had positive PMI indicating over treatment of pain (Figure 1).

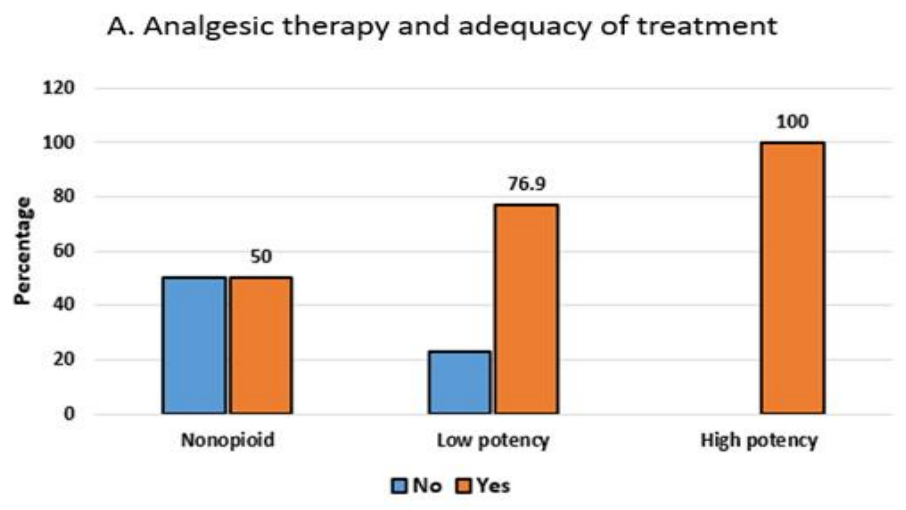

Figure 1: (A). Adequacy of pain management among various analgesic classes and (B). In correlation with pain severity.

There was a statistically significant relationship between PMI and pain severity index $(P=0.007<0.01)$. There was a statistically significant relationship between pain severity index and functional interference index $(\mathrm{P}=$ $0.000<0.01)$.

\section{Factors associated with adequacy of cancer pain management (positive or negative PMI)}

An exploratory univariate analysis of age groups, occupation, level of education and marital status didn't have any effect on the level of pain management. Gender was the only factor that significantly associated with inadequate pain management. Men were more likely to have their pain undermanaged and over managed compared to women.

\section{DISCUSSION}

Cancer associated pain is clearly one of the most distressing symptoms experienced by cancer patients which affects their quality of life. To the best of our knowledge, very little research has examined the adequacy of cancer pain management in Ghana. However, data regarding the adequacy of cancer pain management in cancer directorates in Ghana and the West African sub-region at large is critical to improve cancer pain management. It is our fervent believe that this study will underscore the importance of cancer pain management and provoke a region-wide research to investigate the true state of cancer pain management in sub-Saharan Africa.

Cronbach's alpha coefficients of 0.78 and 0.92 obtained for pain intensity index and functional interference index respectively show that there was excellent internal consistency of all the 4 items under pain intensity index and the 7 items under functional interference index respectively and hence good overall internal consistency of the data collection tool. The results of this study is in agreement with the results of previous studies done to evaluate the Cronbach's $\alpha$ coefficients of pain intensity index and functional interference index components of the BPI in cancer patients in similar settings which ranged from 0.78 to 0.96 and 0.83 to 0.95 respectively. $^{4-6,14}$

There was a statistically significant correlation between pain severity index and functional interference index which is comparable to that reported by other authors in previous studies. ${ }^{5,10,15}$ The moderate correlation between pain severity index and functional interference index reported in this study is different from those reported in other studies where there were stronger or weaker correlations between pain severity index and functional interference index. ${ }^{5,10}$

There exists several practice guidelines designed to facilitate and standardize pharmacologic cancer pain management and advise physicians worldwide on how to achieve optimum cancer pain control. ${ }^{16,17}$ But the most widely used are the guidelines developed by the WHO involving a 3-step analgesic ladder which was employed in this study. According to the WHO ladder algorithm, the selection of non-opioid, weak opioid, strong opioid as well as adjuvant analgesics should be individualized and based on pain intensity. ${ }^{17}$ In this study, strong opioids (e.g. morphine) were frequently used in the management of moderate cancer pain which is contrary to the WHO analgesic ladder stipulations. It is therefore not surprising that majority of the participants in this study were over treated $(\mathrm{PMI}>0)$ for their pain. This practice can lead to 
opioid related tolerance, dependence (physical and psychological) as well as abuse. The results of this study are different from the results of other studies in similar settings where weak opioids (e.g. tramadol) were commonly used for the management of moderate cancer pain as recommended by the WHO. ${ }^{18}$

Pain should be actively managed even when it is mild as it has been reported that pain rated as low as 2 on a 0 -to$10 \mathrm{NRS}$ is severe enough to interfere with the daily functioning of cancer patients. ${ }^{2}$ Although, in this study a relatively small number of respondents $(16 \%)$ had undermanaged cancer pain (PMI <0) compared to the results of other studies where nearly half of the respondents had undermanaged pain, the issue of inadequate cancer pain assessment and management particularly in developing countries is worth considering. ${ }^{9,19}$ Perhaps the recommendations for the assessment and management of cancer pain by the Joint Commission on Accreditation of Healthcare Organizations, an independent non-profit organization in the United States of America can be adopted and utilised for cancer pain assessment and management in accredited hospitals and other healthcare settings in Ghana. ${ }^{2}$ The key recommendations of the Joint Commission on Accreditation of Healthcare Organizations include: proper assessment of the nature and severity of pain in all patients, proper record keeping of the results of pain assessment in a way that facilitates regular reassessment and follow-up, establishment of policies and procedures that support the appropriate prescription or ordering of effective pain medications. ${ }^{2,20}$

The fact that men were more likely to have their pain undermanaged or over-managed is corroborated by a previous study where males were more likely to report inadequate pain management based on PMI scores. ${ }^{9}$ This is however contrary to the results of the study by Edrington et al. where there were no sex differences in the adequacy of pain management based on PMI scores. $^{19,21}$

Overall quality of pain control can be influenced by the patient's attitudes, beliefs and misconceptions about pain management modalities. ${ }^{22}$ Patients should therefore be given adequate instructions at the hospital to correct possible misconceptions about pain treatment (e.g., fear of addiction). Consistent information about cancer pain and pain relief should also be provided along with clear and concise instructions concerning regular pain medication intake, dose adjustments, management of drug side effects and the use of non-pharmacological interventions in the management of cancer pain.

\section{ACKNOWLEDGEMENTS}

Our gratitude goes to all patients who participated in the study as well as the staff at the Oncology Directorate, KATH.
Funding: No funding sources Conflict of interest: None declared

Ethical approval: The study was approved by the Committee on Human Research Publications and Ethics, KNUST, Kumasi, Ghana (CHRPE/RC/012/15)

\section{REFERENCES}

1. Reyes-Gibby CC, Ba Duc N, Phi Yen N, Hoai Nga $\mathrm{N}$, Van Tran T, Guo H et al. Status of cancer pain in Hanoi, Vietnam: A hospital-wide survey in a tertiary cancer treatment center. J Pain Symptom Manage. 2006;31(5):431-9.

2. Okuyama T, Wan, XS, Akechi T, Mendoza TR, Hosaka T, Cleeland CS et al. Adequacy of cancer pain management in a Japanese Cancer Hospital. Jpn J Clin Oncol. 2004;34(1):37-42.

3. Batalha LM, Mota AA. Massage in children with cancer: effectiveness of a protocol. J Pediatr (Rio J). 2013;89(6):595-600.

4. Black B, Herr K, Fine P, Sanders S, Tang X, BergenJackson $\mathrm{K}$ et al. The relationships among pain, nonpain symptoms, and quality of life measures in older adults with cancer receiving hospice care. Pain Med. 2011;12(6):880-9.

5. Ballout S, Noureddine S, Huijer HA, Kanazi G. Psychometric evaluation of the arabic brief pain inventory in a sample of Lebanese cancer patients. J Pain Symptom Manage. 2011;42(1):147-54.

6. Aisyaturridha A, Naing L, Nizar AJ. Validation of the Malay Brief Pain Inventory questionnaire to measure cancer pain. J Pain Symptom Manage. 2006;31(1):13-21.

7. Prommer EE. Pharmacological Management of Cancer-Related Pain. Cancer Control. 2015;22(4):412-25.

8. Wiredu EK, Armah HB. Cancer mortality patterns in Ghana: a 10-year review of autopsies and hospital mortality. BMC public health. 2006;6(1):1.

9. Larue F, Fontaine A, Colleau SM. Underestimation and undertreatment of pain in HIV disease: multicentre study. Bmj. 1997;314(7073):23.

10. Caffo O, Sava T, Comploj E, Fariello A, Zustovich F, Segati $R$ et al. Impact of docetaxel-based chemotherapy on quality of life of patients with castration-resistant prostate cancer: results from a prospective phase II randomized trial. BJU Int. 2011;108(11):1825-32.

11. Eggermont LH, Leveille SG, Shi L, Kiely DK, Shmerling RH, Jones RN et al. Pain characteristics associated with the onset of disability in older adults: the maintenance of balance, independent living, intellect, and zest in the Elderly Boston Study. J Am Geriatr Soc. 2014;62(6):1007-16.

12. Bortsov AV, Platts-Mills TF, Peak DA, Jones JS, Swor RA, Domeier RM et al. Effect of pain location and duration on life function in the year after motor vehicle collision. Pain. 2014;155(9):1836-45.

13. Mitera G, Fairchild A, DeAngelis C, Emmenegger U, Zurawel-Balaura, L, Zhang, L et al. A multicenter 
assessment of the adequacy of cancer pain treatment using the pain management index. J Palliat Med. 2010;13(5):589-93.

14. Atkinson TM, Mendoza TR, Sit L, Passik S, Scher $\mathrm{HI}$, Cleeland $\mathrm{C}$ et al. The Brief Pain Inventory and its pain at its worst in the last 24 hours item: clinical trial endpoint considerations. Pain Med. 2010;11(3):33746.

15. McKee MD, Kligler B, Fletcher J, Biryukov F, Casalaina W, Anderson B et al. Outcomes of acupuncture for chronic pain in urban primary care. $\mathrm{J}$ Am Board Fam Med. 2013;26(6):692-700.

16. Jacox A, Carr DB, Payne R. New clinical-practice guidelines for the management of pain in patients with cancer. N Engl J Med. 1994;330(9):651-5.

17. Benedetti S, Pirola B, Pollo B, Magrassi L, Bruzzone MG, Rigamonti D et al. Gene therapy of experimental brain tumors using neural progenitor cells. Nat Med. 2000;6(4):447-50.

18. Ogboli-Nwasor E, Makama J, Yusufu L. Evaluation of knowledge of cancer pain management among medical practitioners in a low-resource setting. J Pain Res. 2013;6:71-7.
19. Donovan KA, Taliaferro LA, Brock CW, Bazargan S. Sex differences in the adequacy of pain management among patients referred to a multidisciplinary cancer pain clinic. J Pain Symptom Manage. 2008;36(2):167-72.

20. Organizations JCoAoH. Joint Commission on Accreditation of Healthcare Organizations pain standards for 2001. Joint Commission Resources, Inc.

21. Edrington JM, Paul S, Dodd M, West C, Facione N, Tripathy $\mathrm{D}$ et al. No evidence for sex differences in the severity and treatment of cancer pain. J Pain Symptom Manage. 2004;28(3):225-32.

22. Beauregard L, Pomp A, Choiniere M. Severity and impact of pain after day-surgery. Can J Anaesth. 1998;45(4):304-11.

Cite this article as: Abruquah AA, Biney RP, OseiBonsu EB, Boamah KM, Woode E. Adequacy of pain management in oncology patients at a tertiary hospital in Ghana. Int J Basic Clin Pharmacol 2017;6:251-6. 